\title{
An Algorithm for Thermodynamic Parameter Optimization: Application to the Martian mantle
}

\author{
DEAN KHAN, CHRISTIAN LIEBSKE AND JAMES A. D. \\ CONNOLLY
}

Institute of Geochemistry and Petrology

Presenting Author: dean.khan@erdw.ethz.ch

Thermodynamic models for geologically relevant chemical compositions are a valuable resource in predicting the physical and thermochemical properties of planetary interiors. However, predicted stable assemblages may deviate considerably from experimental data and are of particular concern for compositions beyond the range considered during the calibration of the thermodynamic database, as parameters were not directly optimized for these compositions. This creates complications when trying to invert physical properties for a planets thermochemical structure. We have developed a Bayesian algorithm that permits the modification of a thermodynamic model to account for additional observational constraints. To demonstrate its applicability, it is used to revise the Stixrude \& Lithgow-Bertelloni (2011) whole-mantle terrestrial thermodynamic model, using phase equilibrium constraints provided by Bertka \& Fei (1997), for the more iron-rich compositions that are thought to be relevant to the Martian mantle. Our results show that small changes to the thermodynamic dataset result in a marked improvement in the agreement between the predicted and experimental results, providing estimates for geophysical properties that are more consistent with experimental data. Seismic properties are calculated in an internally self-consistent manner along hot and cold areotherms to constrain the upper and lower bounds of these properties for different bulk silicate Mars compositional models. A revised thermodynamic model will improve our ability to infer the composition of the Martian mantle from the seismic data provided by NASA's InSight mission. 